\title{
OPTICAL COHERENCE TOMOGRAPHY ANGIOGRAPHY CHANGES IN MYOPIC CHOROIDAL NEOVASCULARIZATION BEFORE AND AFTER INTRAVITREAL RANIBIZUMAB INJECTION
}

\author{
By
Mohamed Medhat Sakhsoukh, Ahmed M. Youssef and Abd El-Ghany Ibrahim Abd El-Ghany

Ophthalmology Department, Faculty of Medicine, Al Azhar University

Corresponding author: Mohamed Medhat Sakhsoukh,

Phone: 01004491165, E-mail: knight_2210@yahoo.com

\begin{abstract}
Background: Progressive and excessive elongation of the globe may lead to many complication one of them is myopic choroidal neovascularization which can be diagnosed with FFA, OCT and OCTA and treated with intravitreal injection of Ranibizumab.

Objective: To investigate morphological changes in myopic choroidal neovascularization (mCNV) using optical coherence tomography-angiography (OCT-A) after treatment with ranibizumab.

Patients and methods: This study is prospective cross sectional interventional study on 50 high myopic eyes with naive mCNV. All patients were imaged at baseline with color pictures, FFA, OCT and OCT-A at baseline and after treatment.

Results: There was a significant improvement of best corrected visual acuity and decrease in central foveal thickness after intravitreal injection of Ranibizumab. Optical coherence tomography angiography can detect myopic choroidal neovascularization with high sensitivity with different patterns (sea-fan, medusa and treein-bud) but after treatment this abnormal vascularization may not be detected in some cases and lower number of injection needed in tree-in-bud pattern.

Conclusion: OCTA can be used in diagnosis of $\mathrm{mCNV}$ with high sensitivity $(88 \%)$ with three patterns treein-bud, medusa and sea-fan with tree-in-bud represents the most common pattern of mCNV. Ranibizumab intravitreal injection is an effective treatment in $\mathrm{mCNV}$ using $1+\mathrm{PRN}$ protocol with significant improvement of BCVA and decrease in CFT. Unlike CNV due to AMD mCNV may regress with single or double injection with improvement of BCVA and larger number of injection is usually needed in older age patients and with mature patterns (medusa and sea-fan) of mCNV.
\end{abstract}

Keywords: Intravitreal Ranibizumab Injection; Optical Coherence Tomography Angiography; myopia.

Disclosure: The authors have no financial interest to declare in relation to the content of this article.

\section{INTRODUCTION}

High myopia is one of the leading causes of visual impairment in many developed countries. Pathological myopia is defined by an axial length of the eye greater than $26 \mathrm{~mm}$ and by a refractive error of -6 dioptres (D) or more, associated with complications of the posterior segment due to 
progressive and excessive elongation of the globe. Progressive posterior segment elongation is accompanied by degenerative changes, including the sclera, optic disc, choroid, Bruch's membrane, retinal pigment epithelium (RPE) and neural retina. These degenerative changes may lead to the development of macular lesions, such as myopic choroidal neovascularisation (CNV) (OhnoMatsui et al., 2016).

The diagnosis of myopic $\mathrm{CNV}$ is confirmed by fundus fluorescein angiography (FFA) and structural spectral-domain optical coherence tomography (SD-OCT) B-scan. Myopic CNVs are usually type 2 neovascularisations on OCT or 'classic' on FFA, with well-defined hyperfluorescence on early frames and leakage of the dye on late frames. A structural SD-OCT B-scan shows a dome-shaped hyper reflective elevation above the RPE, often associated with discrete retinal changes, including oedema and neurosensory serous retinal detachment (Leveziel et al., 2013).

Optical coherence tomography angiography (OCT-A) is non-invasive imaging technique that employs motion contrast imaging to highresolution volumetric blood flow information generating the same cross-section in order to construct a map of blood flow (Schwartz et al., 2014).

Optical coherence tomography angiography revealed a high-flow neovascular network in $90 \%$ of eyes. OCTA detected 3 different patterns a
Medusa pattern, corresponding to vessels radiating in all directions from the lesion center, Sea-fan pattern, corresponding to vessels radiating in all directions from one side of the lesion and Tree-in-bud pattern, corresponding to small round lesions without obvious vascular trunk. This pattern was the most commonly observed (Cohen, et al., 2019).

Therapeutic modalities for myopic $\mathrm{CNV}$ include laser photo $\neg$ coagulation for extrafoveal and juxtafoveal $\mathrm{CNV}$ and verteporfin photodynamic therapy (PDT) for subfoveal CNV, antivascular endothelium growth factor (Anti-VGEF) and surgical removal of CNV (Fedra et al., 2015).

Based on the results of clinical studies and trials, the anti-VEGF drugs have demonstrated superior efficacy to the traditional modalities in the treatment of $\mathrm{CNV}$ secondary to pathologic myopia. Therefore, intravitreal injection of anti-VEGF drugs has been proposed as the firstline treatment of $\mathrm{CNV}$ secondary to pathologic myopia. Among the antiVEGF drugs, bevacizumab, ranibizumab and aflibercept exhibit similar efficacy in restoring functional and anatomical parameters. However, ranibizumab and aflibercept are designed and approved specifically for ocular administration, and achieves efficacy with shortened duration, frequency, and adverse effects, hence appears to be the first choice for treatment of myopic $\mathrm{CNV}$ when available (Zhang et al., 2015).

The aim of this study was to assess optical coherence tomography 
angiography changes in myopic choroidal neovascularization before and after three on monthly basis intravitreal injection of ranibizumab.

\section{PATIENTS AND METHODS}

Fifty eyes from fifty patients with CNV secondary to high myopia visited the Eye Clinic of Sayed Galal hospital and Kalawon eye hospital between March 2018 and November 2020.

Patients were enrolled in the study according to the following criteria: Treatment-naïve patients suffering from myopic CNV, myopia more than or equal $6 \mathrm{D}$ spherical equivalent and axial length more than or equal $26 \mathrm{~mm}$.

Patients with the following criteria were excluded from the study: Agerelated macular degeneration, media opacity and previous retinal surgery.

The study was approved by Institutional Review Board of the Faculty of Medicine, Al-Azhar University and informed consent was obtained from all subjects before enrollment. All investigations complied with the principles of the Declaration of Helsinki (64 ${ }^{\text {th }}$ WMA General Assembly, Fortaleza, Brazil, October 2013).

All patients studied underwent complete ophthalmic examination, including best-corrected visual acuity (BCVA), using log MAR charts, slit-lamp examination, fundus biomicroscopy. Ultrasound A- scan (PAC Scan, Sonomed, Carleton Optical, Buckinghamshire, UK). SD-OCT (AngioVue, RTVue XR Avanti, Optovue, Inc., Freemont, CA, USA) at baseline and after regression of $\mathrm{CNV}$ or after 3 injections which is nearer. OCT-A
$3 \times 3 \mathrm{~mm}$ macular scan (AngioVue, RTVue XR Avanti, Optovue, Inc., Freemont, CA, USA) at baseline and after regression of $\mathrm{CNV}$ or after 3 injection which is nearer. Fundus colored image and FA (ZEISS VISUCAM 224, Carl Zeiss Meditec, Dublin, CA, USA) at baseline.

All patients were treated with intravitreal injection of ranibizumab $(0.5$ mg) with a $1+$ PRN regimen (single injection then assessing the activity with OCTA and OCT and reinjection only if there is activity) with maximum 3 injections on monthly basis.

Patients in the study are subdivided into 3 comparative groups according to the pattern of mCNV on OCTA.

The primary outcome measures were changes in terms of BCVA in log MAR, changes central foveal thickness, subretinal fluid and cystic changes in SDOCT and membrane morphology and sign of activity in in the three OCTA patterns groups. The secondary outcomes were the average number of injections needed during the follow-up.

\section{Statistical Analysis:}

Data were collected, revised, coded and entered to the Statistical Package for the Social Science (IBM SPSS) version 23. The quantitative data were presented as mean, standard deviations and ranges. Also qualitative variables were presented as number and percentages. The comparison between groups regarding qualitative data was done by using Chisquare test and/or Fisher exact test when the expected count in any cell found less than 5. The comparison between two independent groups with quantitative data 
with parametric distribution was done by using Independent t-test. While the comparison between two independent groups with quantitative data with nonparametric distribution was done by using Mann-Whitney test while more than two groups were done by using KruskallWallis test. The comparison between two paired groups with quantitative data with parametric distribution was done by using Paired t-test. Spearman correlation coefficients were used to assess the correlation between two quantitative parameters in the same group. The confidence interval was set to $95 \%$ and the margin of error accepted was set to $5 \%$.

\section{RESULTS}

Fifty patients 36 females (72.0\%), 14 males $(28.0 \%)$, mean age $56.60 \pm 14.66$ years) for a total 50 eyes $30(60.0 \%)$ right eyes, 20 (40\%) left eyes) were enrolled in this prospective study (table 1). Mean axial length was $28.52 \pm 2.23$. Mean spherical equivalent refraction was -12.72 \pm 4.75 (Table 1).

Table (1): Demographic, characteristics, refraction and axial length of the studied patients

\begin{tabular}{|l|l|c|}
\hline \multicolumn{2}{|l|}{} & Total no. $=50$ \\
\hline \multirow{2}{*}{ Age } & Mean \pm SD & $56.60 \pm 14.66$ \\
\cline { 2 - 3 } & Range & $24-80$ \\
\hline \multirow{2}{*}{ Sex } & Females & $36(72.0 \%)$ \\
\cline { 2 - 3 } & Males & $14(28.0 \%)$ \\
\hline \multirow{2}{*}{ Refraction } & OD & $30(60.0 \%)$ \\
\cline { 2 - 3 } & OS & $20(40.0 \%)$ \\
\hline \multirow{2}{*}{ Axial length } & Mean \pm SD & $12.72 \pm 4.75$ \\
\cline { 2 - 3 } & Range & $6-20$ \\
\cline { 2 - 3 } & Mean \pm SD & $28.52 \pm 2.23$ \\
\cline { 2 - 3 } & Range & $26-33$ \\
\hline
\end{tabular}

Optical coherence tomography angiography (OCTA) detected myopic CNV in 44 of the 50 eyes with sensitivity $88.0 \%$. All CNV appeared in outer retinal layer in OCTA (Figure 1, 2). Artifacts noticed in $4(8.0 \%)$ eyes in the form of motion projection and segmentation artifacts. At baseline, CNV were classified into tree-in-bud $22(50 \%)$, Medusa 12 (27.3\%), or sea-fan $10(22.7 \%) \mathrm{CNV}$. 


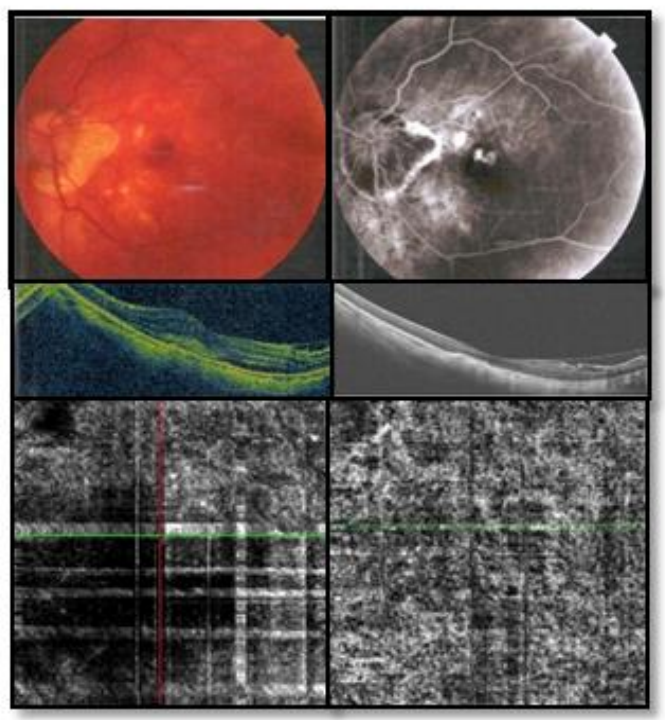

Figure (1): Patient with not detected CNV in OCTA: left eye of 65 years male; color photo of the patient (upper left) FFA angiography in late phase showing leakage of $\mathrm{mCNV}$ (upper right) OCT showing activity of $\mathrm{mCNV}$ and SRF CFT $=240 \mu \mathrm{m}$ (middle left) OCT after 3 injections showing resolution of SRF CTF $=200 \mu \mathrm{m}$ (middle right) choriocapillaris layer of OCTA with no detection of $\mathrm{mCNV}$ with motion artifact (lower left) OCTA after treatment showing no detection of any vascular abnormality but with less artifact (lower right)

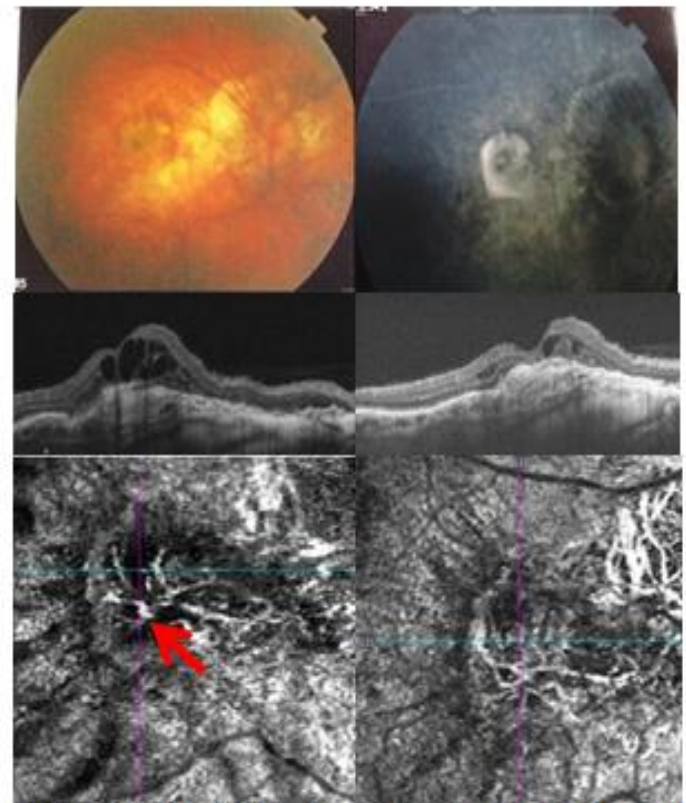

Figure (2): Patient with still active CNV after 3 injection(resistant): Right eye of 54 years old male; color photo (upper left) FFA showing leakage in late stage (upper right) OCT pre injection showing active $\mathrm{mCNV}$ with cystoid macular edema (middle left) OCT after 3 injections showing still active CNV but withless edema (middle right) OCTA pre injection showing CNV in the form of seafan with vascular core (red arrow) (lower left) OCTA after 3 injection showing CNV in the form of Seafan but the membrane become more obvious after decrease in edema (lower right). 
After injections, no signs of activity were observed in OCT and OCTA except in 2 eyes showed still active CNV (figure 4) and there is no change in pattern showed in $24(54.5 \%)$ eyes and change in pattern in $20(45.5 \%)$ eyes with highly statistically significant difference found between OCTA pre and post injection with $\mathrm{p}$-value $=<0.001$. After injection 15 $(34.1 \%)$ eyes showed disappearance of any vascular abnormal membrane, tree inbud, medusa and sea-fan $14(31.82 \%)$ eyes, $11(25.0 \%)$ eyes and $4(9.1 \%)$ eyes respectively (Table 2).

Table (2): OCTA results pre and post injection shows that there was highly statistically significant difference found between OCTA pre and post injection with p-value $<0.001$

\begin{tabular}{|c|c|c|c|}
\hline OCTA & Pre & Post & \multirow{2}{*}{ P-value* } \\
\hline Negative & $0(0.0 \%)$ & $16(36.4 \%)$ & \multirow{2}{*}{$<0.001$} \\
\hline Seafan & $10(22.7 \%)$ & $6(13.6 \%)$ & \\
\hline Medusa & $12(27.3 \%)$ & $6(13.6 \%)$ & \\
\hline Bud in tree & $22(50.0 \%)$ & $16(36.4 \%)$ & \\
\hline
\end{tabular}

*: Fisher exact test

Comparing the three patterns of $\mathrm{CNV}$ groups in OCTA there is non-significant difference between the 3 groups regarding age, eye laterality, refraction and axial length of studied eyes (P-value $>0.05)$ but there is a significant difference regarding sex as no medusa pattern appeared in males (Table 3).

Table (3): Difference in the 3 groups according to demographic and refractive properties of the studied eyes

\begin{tabular}{|c|c|c|c|c|c|}
\hline \multirow{2}{*}{\multicolumn{2}{|c|}{$\begin{array}{l}\text { OCTA patterns } \\
\text { Parameters }\end{array}$}} & Seafan & Medusa & Bud in tree & \multirow{2}{*}{ P-value } \\
\hline & & No. $=10$ & No. $=12$ & No. $=22$ & \\
\hline \multirow{2}{*}{ Age } & Mean \pm SD & $53.00 \pm 10.18$ & $60.50 \pm 6.60$ & $56.73 \pm 18.65$ & \multirow{2}{*}{$0.490 \bullet$} \\
\hline & Range & $35-64$ & $51-70$ & $24-80$ & \\
\hline \multirow{2}{*}{ Sex } & Females & $6(60.0 \%)$ & $12(100.0 \%)$ & $14(63.6 \%)$ & \multirow{2}{*}{$0.044^{*}$} \\
\hline & Males & $4(40.0 \%)$ & $0(0.0 \%)$ & $8(36.4 \%)$ & \\
\hline \multirow{2}{*}{ Eye } & OD & $4(40.0 \%)$ & $8(66.7 \%)$ & $16(72.7 \%)$ & \multirow{2}{*}{$0.197 *$} \\
\hline & OS & $6(60.0 \%)$ & $4(33.3 \%)$ & $6(27.3 \%)$ & \\
\hline \multirow{2}{*}{ Refraction } & Mean \pm SD & $14.00 \pm 4.94$ & $14.17 \pm 3.69$ & $11.00 \pm 4.43$ & \multirow{2}{*}{$0.086 \neq$} \\
\hline & Range & $6-20$ & $10-20$ & $6-20$ & \\
\hline \multirow{2}{*}{ Axial length } & Mean \pm SD & $29.55 \pm 2.43$ & $30.11 \pm 2.23$ & $27.35 \pm 1.55$ & \multirow{2}{*}{$0.196^{\bullet}$} \\
\hline & Range & $27-32$ & $27-33$ & $25-30$ & \\
\hline
\end{tabular}

*: Chi-square test; $\bullet:$ One Way ANOVA test; $\neq$ : Kruskal-Wallis test

The mean intravitreal injections number was $2.60 \pm 0.70$ (Table 4). No IVT injection side effects occurred except subconjuctival hemorrhage in 4 patients. When Correlating the age with number of injections we found a positive significant correlation which means that larger number of injections were needed in older patients ( $\mathrm{p}$-value $=0.035$ and $\mathrm{r}=0.298$ ). 
Table (4): Number of anti-VGEF injections in studied eyes

\begin{tabular}{|c|c|c|}
\hline \multirow{4}{*}{ Number of injections } & 1 & $6(12.0 \%)$ \\
\cline { 2 - 3 } & 2 & $8(16.0 \%)$ \\
\cline { 2 - 3 } & 3 & $36(72.0 \%)$ \\
\cline { 2 - 3 } & Mean \pm SD & $2.60 \pm 0.70$ \\
\cline { 2 - 3 } & Range & $1-3$ \\
\hline
\end{tabular}

When comparing the three OCTA pattern group there is a significant difference in the number of injections needed in each group with less number of injections needed in the bud in tree group and no patient in sea-fan and medusa group treated with single injection but there is non-significant difference in the mean numbers which are $2.36 \pm 0.90,2.83$ \pm 0.39 and $2.60 \pm 0.52$ in bud in tree, medusa and sea-fan respectively (Table 5).

Table (5): Difference in number of injection between studied groups

\begin{tabular}{|c|c|c|c|c|c|}
\hline \multirow{2}{*}{\multicolumn{2}{|c|}{$\begin{array}{ll}\text { OCTA patterns } \\
\text { Parameters }\end{array}$}} & Seafan & Medusa & Bud in tree & \multirow{2}{*}{$\begin{array}{c}\mathbf{P}- \\
\text { value }\end{array}$} \\
\hline & & No. $=10$ & No. $=12$ & No. $=22$ & \\
\hline \multirow{3}{*}{ Number of injection } & 1 & $0(0.0 \%)$ & $0(0.0 \%)$ & $6(27.3 \%)$ & \multirow{3}{*}{0.037} \\
\hline & 2 & $4(40.0 \%)$ & $2(16.7 \%)$ & $2(9.1 \%)$ & \\
\hline & 3 & $6(60.0 \%)$ & $10(83.3 \%)$ & $14(63.6 \%)$ & \\
\hline \multirow{2}{*}{ Number of injection } & Mean \pm SD & $2.60 \pm 0.52$ & $2.83 \pm 0.39$ & $2.36 \pm 0.90$ & \multirow{2}{*}{$0.322 \neq$} \\
\hline & Range & $2-3$ & $2-3$ & $1-3$ & \\
\hline
\end{tabular}

P-value > 0.05: Non significant; P-value < 0.05: Significant; P-value < 0.01: Highly significant*: Chi-square test; $\neq$ : Kruskal-Wallis test

The mean BCVA at baseline was 0.94 $\pm 0.34 \log$ MAR and, after injection, it was $0.66 \pm 0.38 \log M A R$ and the difference in BCVA between baseline and after injections shows statistically significant improvement ( $\mathrm{p}$-value $<0.001$ ).
In relation to BCVA in three groups there is non-significant difference in preinjection VA, post-injection VA and VA difference between pre and post injection (Table 6).

Table (6): VA difference between studied group

\begin{tabular}{|l|l|c|c|c|c|}
\hline \multirow{2}{*}{ Parameters } & OCTA patterns & Sea-fan & Medusa & Tree-in-bud & \multirow{2}{*}{$\begin{array}{c}\text { P- } \\
\text { value } \neq\end{array}$} \\
\cline { 3 - 6 } Pre VA & Mean \pm SD & $0.90 \pm 0.35$ & $0.80 \pm 0.23$ & $0.97 \pm 0.38$ & \multirow{2}{*}{0.355} \\
\cline { 2 - 5 } & Range & $0.5-1.3$ & $0.5-1$ & $0.3-1.4$ & \\
\hline \multirow{2}{*}{ Post VA } & Mean \pm SD & $0.62 \pm 0.23$ & $0.57 \pm 0.24$ & $0.65 \pm 0.48$ & \multirow{2}{*}{0.873} \\
\cline { 2 - 5 } & Range & $0.4-1$ & $0.3-1$ & $0.1-1.3$ & \\
\hline \multirow{3}{*}{ VA difference } & Mean \pm SD & $-0.28 \pm 0.41$ & $-0.23 \pm 0.32$ & $-0.32 \pm 0.29$ & \multirow{3}{*}{0.751} \\
\cline { 2 - 5 } & Range & $-0.8-0.3$ & $-0.7-0.2$ & $-0.9-0$ & \\
\cline { 2 - 5 } & Still active & $0(0.0 \%)$ & $1(8.3 \%)$ & $0(0.0 \%)$ & \\
\hline
\end{tabular}

\#: Kruskal-Wallis test 
At baseline, FFA revealed leakage of the lesion in all eyes. Specteral domain optical coherence tomography (SD-OCT) showed subretinal fluid in 38 eyes $(76.0 \%)$ and intra retinal cysts in 18 eyes $(36.0 \%)$ (Table 7). The mean central foveal thickness (CFT) at base line was
$280.52 \pm 72.24 \mu \mathrm{m}$, after injections it was $208.48 \pm 60.15$ and the difference between CFT showed statistically significant decrease in the thickness ( $\mathrm{p}$-value $<0.001$ ) (Table 8). After injection, SD-OCT showed resolution of active signs of $\mathrm{CNV}$ except in 2 eyes.

Table (7): Percentage of patients with SRF and cyst among the studied eyes

\begin{tabular}{|c|c|c|}
\hline \multirow{2}{*}{ SRF } & No & Total no. $=50$ \\
\cline { 2 - 3 } & Yes & $12(24.0 \%)$ \\
\hline \multirow{2}{*}{ Cyst } & No & $38(76.0 \%)$ \\
\cline { 2 - 3 } & Yes & $32(64.0 \%)$ \\
\hline
\end{tabular}

There was a statistically significant decrease in the thickness post injection than pre injection with p-value $<0.001$ (Table 8).

Table (8): Comparison between CFT thickness pre and post injection among the studied patients

\begin{tabular}{|c|c|c|c|c|}
\hline Thickness & Pre & Post & Difference & P-value \\
\hline Mean \pm SD & $280.52 \pm 72.24$ & $208.48 \pm 60.15$ & $-72.04 \pm 68.56$ & \multirow{2}{*}{0.000} \\
\cline { 1 - 4 } Range & $150-430$ & $80-350$ & $-310-30$ & \\
\hline
\end{tabular}

$\bullet:$ Paired t-test

There was a statistically significant negative correlation found between age and thickness difference between pre and post injection that means that thickness difference has been found to be greater among older age group showed statistically significant negative correlation found between age and thickness difference between pre and post while no statistically significant correlation found with the other parameters. Also VA difference between pre and post did not show any correlation with age, refraction and number of injection (Table 9). Also there is statistically significant increase in the difference of VA in cases with SRF and also statistically significant decrease in $\mathrm{VA}$ difference in cases with cyst ( $\mathrm{p}$-value $=0.031$ and $<0.001$ respectively) in studied eyes (Table 10).

Table (9): Correlation of the VA and thickness difference between pre and post injections with age, refraction and number of injection

\begin{tabular}{|l|c|c|c|c|}
\hline \multirow{2}{*}{ Variables } & \multicolumn{2}{c|}{ VA difference } & \multicolumn{2}{c|}{ Thickness difference } \\
\cline { 2 - 5 } & $\mathbf{r}$ & P-value & r & P-value \\
\hline VA difference & - & - & 0.065 & 0.653 \\
\hline Thickness difference & 0.065 & 0.653 & - & - \\
\hline Age & 0.207 & 0.149 & $\mathbf{- 0 . 4 5 7 * *}$ & $\mathbf{0 . 0 0 1}$ \\
\hline Refraction & -0.239 & 0.095 & -0.268 & 0.059 \\
\hline Number of injection & -0.054 & 0.707 & -0.047 & 0.744 \\
\hline
\end{tabular}


Table (1): Relation between VA difference between pre and post with the other parameters of the studied patients

\begin{tabular}{|c|c|c|c|c|}
\hline \multicolumn{2}{|c|}{$\begin{array}{ll}\text { Parameters } & \text { VA difference } \\
\end{array}$} & \multirow{2}{*}{$\begin{array}{c}\text { Mean } \pm \text { SD } \\
-0.32 \pm 0.37\end{array}$} & \multirow{2}{*}{$\begin{array}{c}\text { Range } \\
-0.9-0.3\end{array}$} & \multirow{3}{*}{$\begin{array}{c}\text { P-value } \\
0.337 \bullet\end{array}$} \\
\hline \multirow{2}{*}{ Sex } & Females & & & \\
\hline & Males & $-0.21 \pm 0.22$ & $-0.6-0$ & \\
\hline \multirow{2}{*}{ Eye } & OD & $-0.31 \pm 0.31$ & $-0.9-0.2$ & \multirow{2}{*}{$0.357 \bullet$} \\
\hline & OS & $-0.25 \pm 0.37$ & $-0.9-0.3$ & \\
\hline \multirow{3}{*}{$\begin{array}{l}\text { Number of } \\
\text { injection }\end{array}$} & 1 & $-0.13 \pm 0.14$ & $-0.3-0$ & \multirow{3}{*}{$0.426 \neq$} \\
\hline & 2 & $-0.38 \pm 0.46$ & $-0.8-0.3$ & \\
\hline & 3 & $-0.29 \pm 0.33$ & $-0.9-0.2$ & \\
\hline \multirow{2}{*}{ SRF } & No & $-0.1 \pm 0.26$ & $-0.5-0.3$ & \multirow{2}{*}{$0.031 \bullet$} \\
\hline & Yes & $-0.35 \pm 0.34$ & $-0.9-0.2$ & \\
\hline \multirow{2}{*}{ Cyst } & No & $-0.43 \pm 0.31$ & $-0.9-0$ & \multirow{2}{*}{$<0.001 \bullet$} \\
\hline & Yes & $-0.04 \pm 0.22$ & $-0.5-0.3$ & \\
\hline \multirow{2}{*}{ Artifact } & $\mathrm{No}$ & $-0.3 \pm 0.34$ & $-0.9-0.3$ & \multirow{2}{*}{$0.386^{\bullet}$} \\
\hline & Yes & $-0.15 \pm 0.17$ & $-0.3-0$ & \\
\hline
\end{tabular}

$\bullet$ Mann-Whitney test; $\neq$ : Kruskal-Wallis test

Comparing the studied groups there is a significant difference between the 3 groups in relation to pre injection CFT with the highest thickness in the medusa group (mean thickness $=299.83 \pm 75.98$ ) and highly significant difference in the post injection CFT but non-significance in CFT difference, presence of cyst and SRF (Table 11).

Table (2): SD-OCT parameters difference in studied group

\begin{tabular}{|c|c|c|c|c|c|}
\hline \multirow{2}{*}{\multicolumn{2}{|c|}{$\begin{array}{l}\text { OCTA patterns } \\
\text { Parameters }\end{array}$}} & \multirow{3}{*}{$\begin{array}{c}\text { Seafan } \\
\text { No. }=\mathbf{1 0} \\
225.40 \pm 67.86\end{array}$} & \multirow{2}{*}{$\begin{array}{l}\text { Medusa } \\
\text { No. }=12 \\
\end{array}$} & \multirow{2}{*}{$\begin{array}{c}\text { Bud in tree } \\
\text { No. }=22\end{array}$} & \multirow[b]{2}{*}{ P-value } \\
\hline & & & & & \\
\hline \multirow{2}{*}{ Pre thick } & Mean \pm SD & & $299.83 \pm 75.98$ & $288.18 \pm 64.83$ & \multirow{2}{*}{$0.031 \bullet$} \\
\hline & Range & $150-320$ & $222-424$ & $230-430$ & \\
\hline \multirow{2}{*}{ Post thick } & Mean \pm SD & $166.00 \pm 65.86$ & $258.17 \pm 68.49$ & $195.73 \pm 38.83$ & \multirow{2}{*}{$0.001 \bullet$} \\
\hline & Range & $80-220$ & $170-350$ & $120-270$ & \\
\hline \multirow{2}{*}{$\begin{array}{l}\text { Thickness } \\
\text { difference }\end{array}$} & Mean \pm SD & $-59.40 \pm 25.85$ & $-41.67 \pm 43.32$ & $-92.45 \pm 89.86$ & \multirow{2}{*}{$0.287 \neq$} \\
\hline & Range & $-100--30$ & $-94-30$ & $-310-30$ & \\
\hline \multirow{2}{*}{ SRF } & No & $2(20.0 \%)$ & $2(16.7 \%)$ & $8(36.4 \%)$ & \multirow{2}{*}{$0.394 *$} \\
\hline & Yes & $8(80.0 \%)$ & $10(83.3 \%)$ & $14(63.6 \%)$ & \\
\hline \multirow{2}{*}{ Cyst } & No & $4(40.0 \%)$ & $8(66.7 \%)$ & $16(72.7 \%)$ & \multirow{2}{*}{$0.197 *$} \\
\hline & Yes & $6(60.0 \%)$ & $4(33.3 \%)$ & $6(27.3 \%)$ & \\
\hline \multicolumn{6}{|c|}{ Post hoc analysis by LSD } \\
\hline & & $\begin{array}{l}\text { Seafan Vs } \\
\text { Medusa }\end{array}$ & \multicolumn{2}{|c|}{ Seafan Vs Bud in tree } & $\begin{array}{l}\text { Medusa Vs } \\
\text { Bud in tree }\end{array}$ \\
\hline \multicolumn{2}{|l|}{ Pre thick } & 0.015 & \multicolumn{2}{|c|}{0.021} & 0.639 \\
\hline \multicolumn{2}{|l|}{ Post thick } & 0.000 & \multicolumn{2}{|c|}{0.161} & 0.003 \\
\hline
\end{tabular}




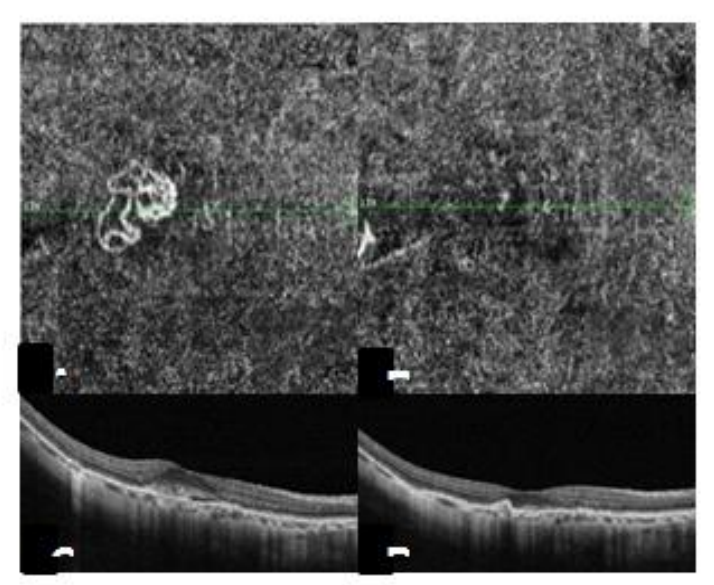

Figure (3): A case with tree-in-bud disappeared after single injection (A) CNV with tree-in-bud pattem in outer retinal layer of $3 \times 3$ OCTA before injection with no visible vascular core nor halo (B) patient $\mathrm{A}$ after single injection shows disappearance of the membrane (C,D) SD-OCT pre and post injection showing disappearance of SRF.

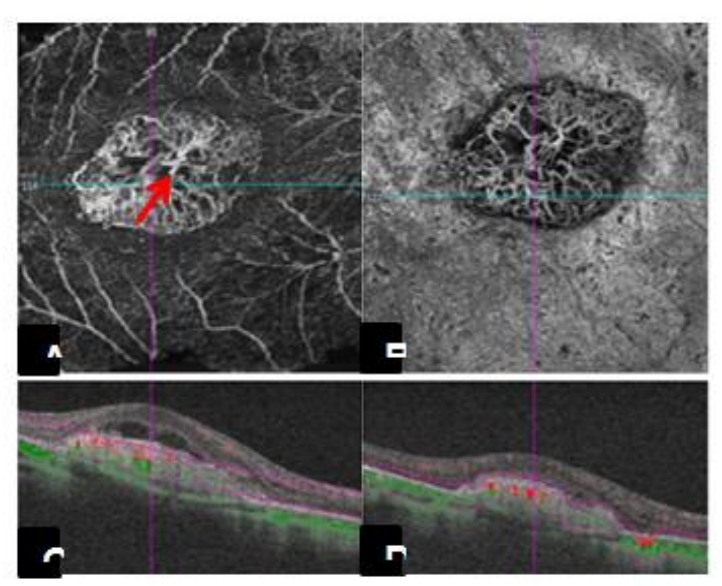

Figure (5):A case with medusa pattem remained medusa after 3 injection (A) $3 \times 3$ OCTA showing medusa pattem $\mathrm{mCNV}$ with vascular core (red arrow) and halo (B) OCTA after injection become inactive with disappearance of vascular core and loss of thin capillaries architecture (C,D) OCT before and after injection showing disappeared SRF.

\section{DISCUSSION}

This study was a prospective cross sectional interventional study on 50 high myopic eyes with naive $\mathrm{mCNV}$ visited the eye clinic of Sayed Galal hospital and

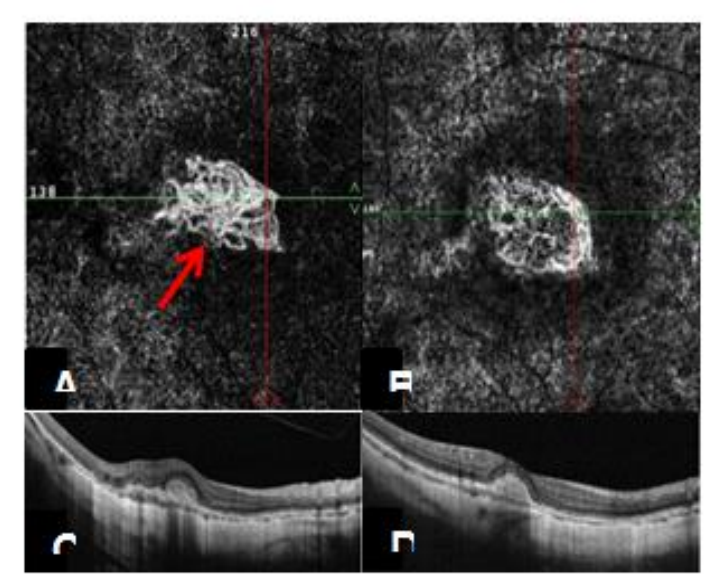

Figure (4): A case with Medusa pattern changed to tree-in-bud after 3 injection (A) OCTA $3 \times 3$ showing nedusa pattern before injections with prei- lesion halo and vascular core (red arrow) (B) patient after 3 injection converted to tree-inbud pattem (C) OCT before injection showing SRF and intra-retinal cysts (D) OCT after injection showing disappearance of activity signs.

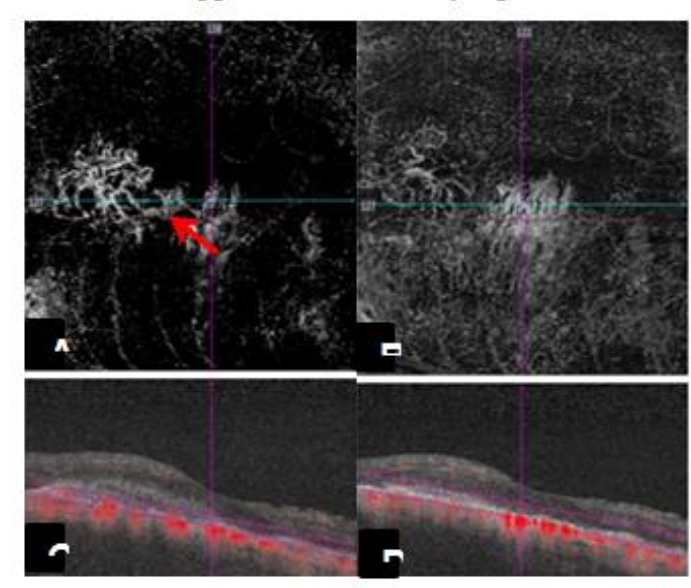

Figure (6): A case of sea-fan $\mathrm{mCNV}$ remained sea-fan after 2 injections (A) $3 \times 3$ OCTA of $\mathrm{mCNV}$ with sea-fan pattem with vascular core (red arrow) (B) OCTA still has the sea-fan pattem after regression with disappeared core (C,D) OCT before and after injection showing disappearance of SRF.

Kalawon eye hospital between March 2018 and November 2020. This study aimed to compare OCTA and OCT finding in myopic CNV before and after injection of Ranibizumab with maximum 3 doses obeying 1+ PRN protocol and 
comparing the 3 resulted patterns group in OCTA regarding BCVA, OCT and number of injections needed in each group.

OCTA detected $44 \mathrm{mCNV}$ of total 50 $\mathrm{mCNV}$ showed leakage in FFA and sign of activity in OCT with $88.0 \%$ sensitivity which is comparable to results of Bruyère et al. (2017) and Querques et al. (2017) that informed $90.48 \%$ and $90 \%$ sensitivity respectively but in comparable to Bagchi et al. (2019) that informed $74.07 \%$.

In systemic review of literature done by Wang et al. (2019), there is a wide range of sensitivities in different studies. These differences in sensitivity may be due to the quality of acquisition of OCTA images in different machines and different numbers of patients in each study (Miyata et al., 2016).

The relative high sensitivity of OCTA in $\mathrm{mCNV}$ detection makes it a good tool for diagnosis of $\mathrm{mCNV}$ especially when accompanied by SD-OCT. So, FFA can be saved as a diagnostic tool in mCNV only when there are unclear images by the OCTA and OCT compromising. Bagchi et al. (2019) mentioned result that when combined, OCTA and SD-OCT or SDOCT and FFA showed similar higher sensitivities than each modality alone.

At baseline mCNV classified into 3 different patterns tree-in-bud (50\%), Medusa $(27.3 \%)$, or sea-fan $(22.7 \%)$ in OCTA which is comparable with Cohen et al. (2019) study informed that tree-in-bud, medusa and seafan pattern represent $55.1 \%, 31 \%$ and $13.9 \%$ of myopic CNV respectively. Cheng et al. (2019) also informed the tree-in-bud pattern of $\mathrm{mCNV}$ is the most common morphological type $(58.3 \%)$.
Cohen et al. (2019) classified active myopic CNV into 3 different patterns a medusa pattern, corresponding to vessels radiating in all directions from the lesion center, Sea-fan pattern, corresponding to vessels radiating in all directions from one side of the lesion and tree-in-bud pattern, corresponding to small round lesions without obvious vascular trunk. The distinction between medusa and sea-fan lesions may be useless, because the development of $\mathrm{CNV}$ in one or more directions may not correspond to a different type of $\mathrm{CNV}$, but rather to the capability of neovascular branches to grow, depending on the eyeball curvature according to the shape of the staphyloma. Also, the difference of OCTA patterns may only relate to the CNV size. Indeed, most of the tree-in-bud CNV had smallsize $\mathrm{CNV}$.

Bruyère et al. (2017) suggested that there are two active neovascular phenotypes prevailed in in myopic CNV in OCTA: first one is disorganized vascular loops a small network consisting of a convoluted vessel and an overall disorganized aspect with no dark halo or feeder vessels (immature) while the second while the second is a larger, highly organized, interlacing type of membrane bordered by a dark halo, with feeder vessel (immature) in comparison to this study the first one is closer to tree-in-bud while the second is more closer to the other 2 patterns in our study.

After injections, there were $34.1 \%$ cases showing disappearance if any abnormal vascularity. There was no change in pattern showed in $54.5 \%$ eyes and change in pattern in $45.5 \%$ eyes with statistically significant difference found 
between OCTA pre and post injection and tree in-bud, medusa and sea-fan $31.82 \%$ eyes, $25.0 \%$ eyes and $9.1 \%$ eyes respectively, while Cohen et al. (2019) study informed disappearance if any abnormal vascularity in $44.8 \%$, and no change in $41.3 \%$ with tree-in-bud pattern still the more common pattern.

Although after injection with regression of activity sometimes the membrane still has a defined pattern, but it showed the signs of in-active membrane (appearance of large diameter vessels, loss of thin capillaries, prominent anastomoses of vessels, weak appearance of vascular core and peri-lesion halo). These were similar to tangled pattern observed by Querques et al. (2017) which is more obvious in non-tree-in-bud patterns. Also, medusa and sea-fan patterns may be converted to indistinct pattern tree-in-bud but not vice versa.

Patients' mean age in our study comparable to Bruyere et al. (2017), Querques et al. (2017), Bagchi et al. (2019) and Mohsen and Kishk (2019).

Regarding to sex of the patient, the result of the study cleared that more females were affected with myopic CNV compared to males which was comparable to Bruyere et al. (2017), Querques et al. (2017) study, Mohsen and Kishk (2019) study.

This study followed the protocol of $1+$ pro re nata (PRN) with maximum 3 injections of Ranibizumab on monthly basis. 1+ PRN means after the first injection we assess the activity and continue injection if there was still activity but in the protocol injection stopped if myopic CNV become inactive. This protocol of injection was followed for myopic $\mathrm{CNV}$ unlike age related $\mathrm{CNV}$ which has two different protocol of antiVGEF injection both of them starts with 3 successive injections on monthly basis then follow either PRN protocol or treat and extend protocol (Augsburger et al., 2019 and Cennamo et al., 2019).

The mean number of Ranibizumab injection was comparable to Cohen et al. (2019). Giorno et al. (2010) and Cennamo et al. (2019) informed lower mean number of injection. This lower number of injections may be due to the lower number of patients in each study.

When comparing the three OCTA pattern groups, there was a significant difference in the number of injections needed in each group with less number of injections needed in the tree-in-bud group and single injection only treated tree-inbud pattern but does not effective in the other patterns.

This study injected only Ranibizumab as an anti-VGEF in the treatment in myopic CNV. Some studies used another anti -VGEF as Aflibercept and bevacizumab in treatment in myopic CNV. When Cha et al. (2014) compared Bevacizumab to Ranibizumab in treatment of myopic CNV, they found that there is no significant difference between them in improving BCVA and CFT. Korol et al. (2020) also informed that there is no significant difference between Aflibercept and Ranibizimab in improving BCVA in myopic CNV.

The results of this study showed positive correlation between the age with number of injections which means that larger number of injections were needed in older patients which is similar to results of Karagiannis et al. (2017) who showed 
that the younger age group needed lower number of injection that older age group.

In this study there was a significant improvement of BCVA after injection, and no significant difference in preinjection VA, post-injection VA and VA difference between pre and post injection between the studied groups. Comparable result was found in REPAIR study which informed gain of 13.8 letters in BCVA respectively after injection. Also, the RADIANCE study showed that the gain in BCVA was 11.9 letters (Tan and Sadda 2017).

There is non-comparable data from Cennamo et al. (2019) and Cohen et al. (2019) with gain of 8 and 6.3 ETDRS letters respectively which may be explained by lower number of patients in each study which is 20 and 11 respectively compared to the above mentioned studies. Cohen et al. (2019) also explained this difference by being a retrospective study.

There was no relation between difference in BCVA and age of the patients, while Karagiannis et al. (2017) informed that age of the patients was also significantly correlated with the improvement in BCVA after treatment with Ranibizumab, but was not significantly correlated with baseline BCVA before injection.

At baseline, Specteral domain optical coherence tomography (SD-OCT) showed subretinal fluid in $66.0 \%$ and intra retinal cysts in $36.0 \%$ in studied eye which was comparable to Lee et al. (2018) who informed intra retinal cyst in $43.48 \%$, and Cennamo et al. (2019) study informed presence of SRF in $60 \%$.
However, there was non-comparable results according to SRF and intra retinal cyst to our study and to each other as Guichard et al. (2010) informed presence of SRF and intra retinal cyst in $50.0 \%$ of eyes and $55.6 \%$ eye respectively, Also, Lee et al. (2018) informed presence of $\mathrm{SRF}$ in $43.48 \%$ of eyes.

The mean CFT at base line showed a significant decrease in CFT thickness after injection in our study. There was a significant difference between the 3 groups in relation to pre injection CFT with the highest thickness in the medusa group, and significant difference in the post injection CFT. Comparable data obtained in Guichard et al. (2010) and Pasyechnikova et al. (2015) showed mean CFT decreased Ranibizumab injection.

Significant negative correlation was found between age and CFT difference pre and post injection. That means that thickness difference has been found to be greater among older age group in this study. This may be explained by baseline increase in CFT in older age group.

Introini et al. (2012) showed that no effect of the presence of baseline sub or intraretinal fluid on visual outcome after treatment unlike to this study that showed statistically significant increase in the difference of VA in cases with SRF as that prove the effect of SRF on VA also this study showed statistically significant decrease in VA difference in cases with cyst.

\section{CONCLUSION}

OCTA can be used in diagnosis of mCNV with high sensitivity ( $88 \%$ ) with three patterns tree-in-bud, medusa and sea-fan with tree-in-bud represents the 


\section{MOHAMED M. SAKHSOUKH et al.,}

most common pattern of mCNV. Ranibizumab intravitreal injection is an effective treatment in mCNV using $1+\mathrm{PRN}$ protocol with significant improvement of BCVA and decrease in CFT. Unlike CNV due to AMD mCNV may regress with single or double injection with improvement of BCVA and larger number of injection is usually needed in older age patients and with mature patterns (medusa and sea-fan) of mCNV.

\section{REFERENCES}

1. Augsburger $M$, Sarra $G$ and Imesch $P$ (2019): Treat and extend versus pro re nata regimens of ranibizumab and aflibercept in neovascular age-related macular degeneration: a comparative study. Graefes Arch Clin Exp Ophthalmol., 257: 1889-95.

2. Bagchi A, Schwartz R, Hykin $P$ and Sivaprasad S (2019): Diagnostic algorithm utilizing multimodal imaging including optical coherence tomography angiography for the detection of myopic choroidal neovascularization. Eye, 33:1111-8.

3. Bruyère E, Miere A, Cohen SY, Martiano D, Sikorav A, Popeanga A, Semoun O, Querques $G$ and Souied EH (2017): Neovascularization secondary to high myopia imaged by optical coherence tomography angiography. Retina, 37:2095-101.

4. Cennamo G, Amoroso F, Schiemer S, Velotti $N$, Alfieri $M$ and de Crecchio $G$ (2019): Optical coherence tomography angiography in myopic choroidal neovascularization after intravitreal ranibizumab. Eur J Ophthalmol., 29(2):239-43.

5. Cha DM, Kim TW, Heo JW, Woo SJ, Park KH, Yu HG and Chung H (2014): Comparison of 1-year therapeutic effect of ranibizumab and bevacizumab for myopic choroidal neovascularization: a retrospective, multicenter, comparative study. BMC Ophthalmol., 21(4);14:69.

6. Cheng Y, Li Y, Huang X and Qu Y (2019): Application of optical coherence tomography angiography to assess anti-vascular endothelial growth factor therapy in myopic choroidal neovascularization. Retina, 39(4):712-8.

7. Cohen SY, Tabary S, El Ameen A, Mrejen S, Quentel $G$ and Giocanti-Auregan A (2019): Vascular remodeling of choroidal neovascularization in older myopic patients treated with ranibizumab. Graefes Arch Clin Exp Ophthalmol., 257: 485-93.

8. Fedra K, Leila $E$ and Ahmed C (2015): Current and emerging treatment options for myopic choroidal neovascularization. Clinical Ophthalmology, 9:733-44.

9. Giorno P, Iacono P, Scarinci F, Di Renzo A, Varano $M$ and Parravano $M$ (2020): Microvasculature Changes of Myopic Choroidal Neovascularization and the Predictive Value of Feeder Vessel Disappearance after Ranibizumab Treatment Revealed Using Optical Coherence Tomography Angiography. Ophthalmologica, 243:263-70.

10. Guichard MM, Peters G, Tuerksever C, Pruente C and Hatz K (2020): Outcome Predictors of SD-OCT-Driven Intravitreal Ranibizumab in Choroidal Neovascularization due to Myopia. Ophthalmologica, 243(2):15462

11. Introini U, Casalino G, Querques G, Gimeno AT, Scotti F and Bandello F (2012): Spectral-domain OCT in anti-VEGF treatment of myopic choroidal neovascularization. Eye (Lond), 26(7):976-82.

12. Karagiannis D, Kontadakis GA, Kaprinis K, Giarmoukakis A, Georgalas I, Parikakis EA and Tsilimbaris MK (2017): Treatment of myopic choroidal neovascularization with intravitreal ranibizumab injections: the role of age. Clin Ophthalmol.,11:1197-201.

13. Korol A, Kustryn T, Zadorozhnyy O, Pasyechnikova N and Kozak I (2020): Comparison of Efficacy of Intravitreal Ranibizumab and Aflibercept in Eyes with Myopic Choroidal Neovascularization: 24Month Follow-Up. Journal of Ocular Pharmacology and Therapeutics: the Official Journal of the Association for Ocular Pharmacology and Therapeutics, 36(2), 122-5. 
14. Lee DH, Kang HG, Lee SC and Kim M (2018): Features of optical coherence tomography predictive of choroidal neovascularisation treatment response in pathological myopia in association with fluorescein angiography. $\mathrm{Br} \mathrm{J}$ Ophthalmol., 102(2):238-42.

15. Leveziel N, Caillaux V, Bastuji-Garin S, Zmuda $M$ and Souied EH (2013): Angiographic and optical coherence tomography characteristics of recent myopic choroidal neovascularization. Am J Ophthalmol., 155(5):913-9.

16. Miyata M, Ooto S, Hata M, Yamashiro K, Tamura H, Akagi-Kurashige Y, Nakanishi H, Ueda-Arakawa N, Takahashi A, Kuroda Y, Wakazono $T$, Yoshikawa $M$ and Yoshimura N (2016): Detection of myopic choroidal neovascularization using optical coherence tomography angiography. Am J Ophthalmol., 165:108-14.

17. Mohsen TA and Kishk HM (2019): Optical coherence tomography angiography in the diagnosis of choroidal neovascular membrane. J Egypt Ophthalmol Soc., 112:108-118.

18. Ohno-Matsui K, Lai TY, Lai CC and Cheung CM (2016): Updates of pathologic myopia. Prog Retin Eye Res., 2016;52:156-87.

19. Pasyechnikova NV, Naumenko VO, Korol AR, Zadorozhnyy OS, Kustryn TB and Henrich PB (2015): Intravitreal ranibizumab for the treatment of choroidal neovascularizations associated with pathologic myopia: a prospective study. Ophthalmologica, 233(1):2-7.
20. Querques L, Giuffrè C, Corvi F, Zucchiatti I, Carnevali A, De Vitis LA, Querques G and Bandello F (2017): Optical coherence tomography angiography of myopic choroidal neovascularisation. $\mathrm{Br} \quad \mathrm{J}$ Ophthalmol., 101(5):609-15.

21. Schwartz DM, Fingler J, Kim DY, Zawadzki RJ, Morse LS, Park SS, Fraser SE and Werner JS (2014): Phase-variance optical coherence tomography: a technique for noninvasive angiography. Ophthalmology, 121:180-7.

22. Tan CS and Sadda SR (2017): Anti-vascular endothelial growth factor therapy for the treatment of myopic choroidal neovascularization. Clin Ophthalmol., 11:17416.

23. Wang $R$, Liang $Z$ and Liu $X$ (2019): Diagnostic accuracy of optical coherence tomography angiography for choroidal neovascularization: a systematic review and meta-analysis. BMC Ophthalmol; 19(1):16271.

24. Zhang Y, Han Q, Ru Y, Bo Q and Wei RH (2015): Anti-VEGF treatment for myopic choroid neovascularization: from molecular characterization to update on clinical application. Drug Des Devel Ther., 9:3413-21. 


\section{التصوير الو عائى المقطعى البصرى المثر ابط فى حالات التوعى الدموى المشيمى الناتج عن قصر النظر قبل وبعد وبعد الحقن داخل الجسم الزجاجى بمادة (ر انيبيزو ماب) محمد مدحت سخسوخ , احمد محمد يوسف، عبد الغنى ابراهيم عبد الغنى قسم طب وجراحة العيون}

E-mail: knight 2210@yahoo.com

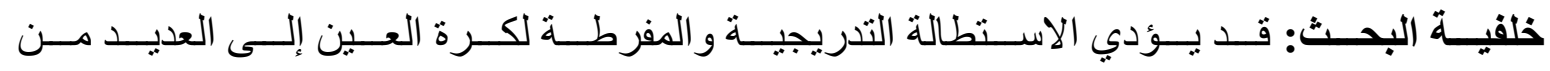

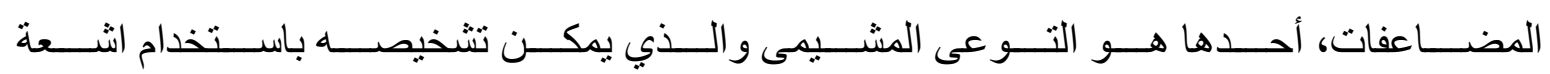

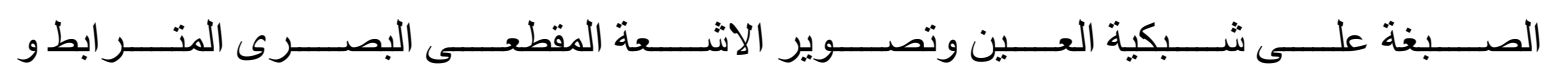

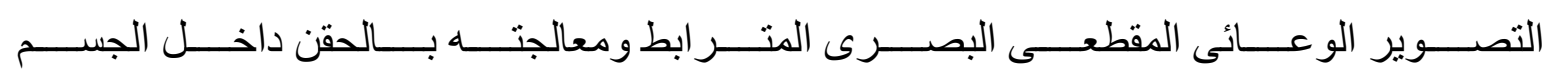
الزجاجي بمادة المضادة لنمو الخلايا المبطنة للاو عية الدموية (رانيبيزوماب).

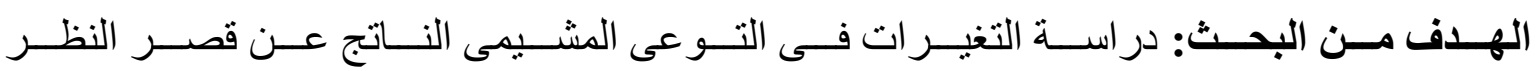

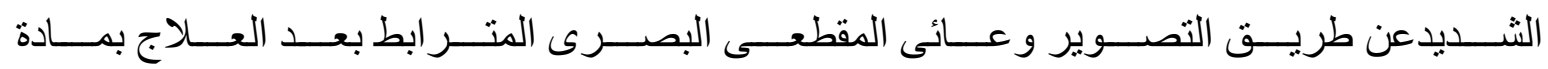
(رانيبيزوماب).

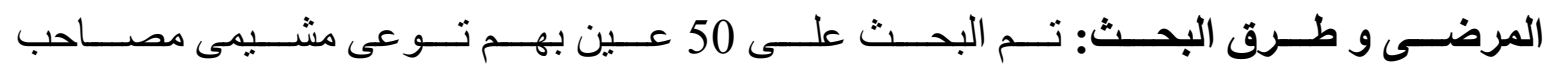

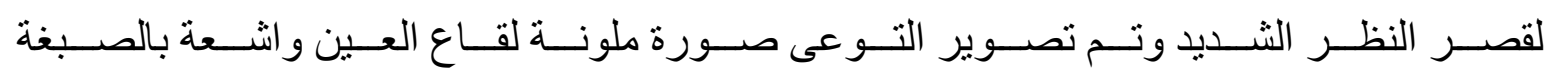

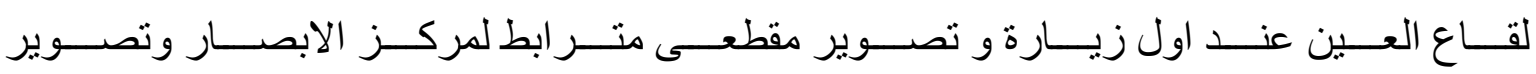
مقطعى و عائى البصرى المتر ابط عند اول زيارة وبعد العلاج.

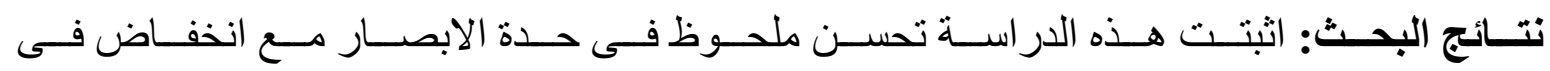

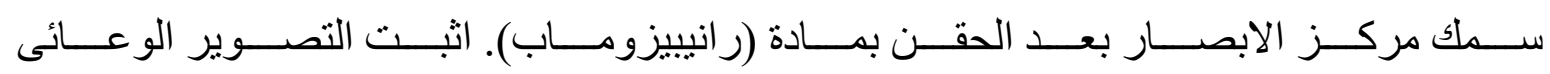

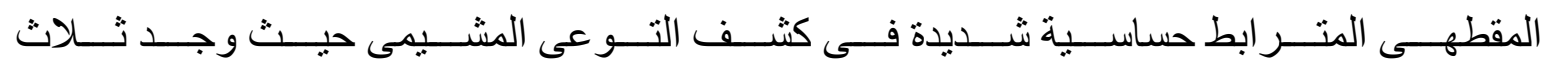

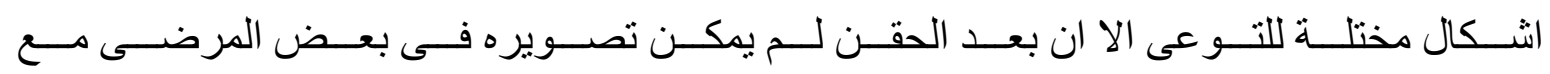
اقل عدد من الحقنات فى الثكل الثجيرى.

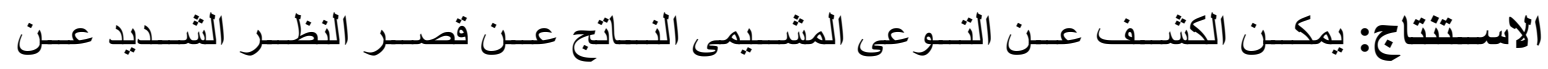

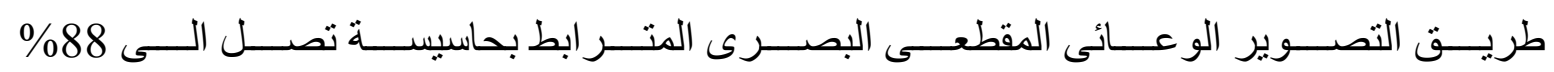




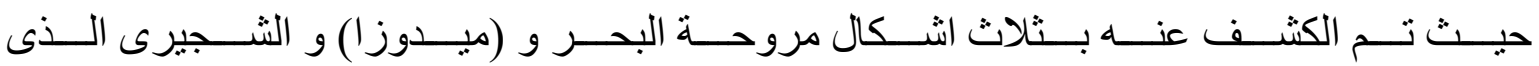

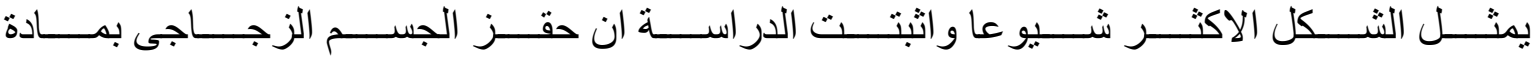

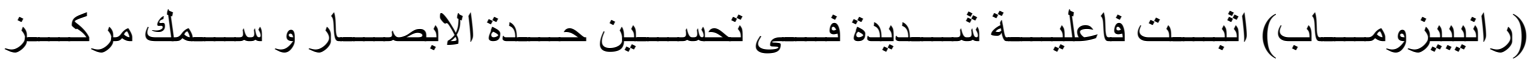

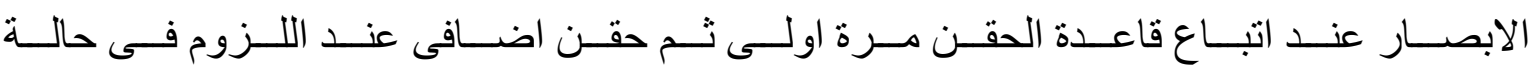

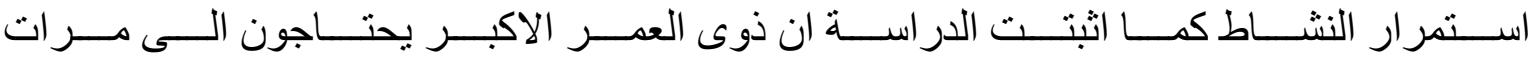

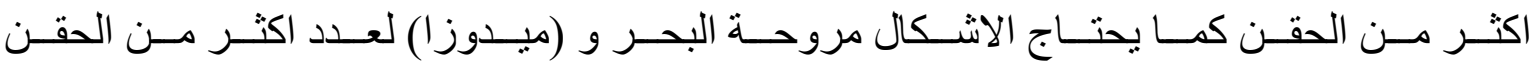
مقارنة بالشكل الثجيرى للتوعى المشيمى الناتج عن قصر النظر.

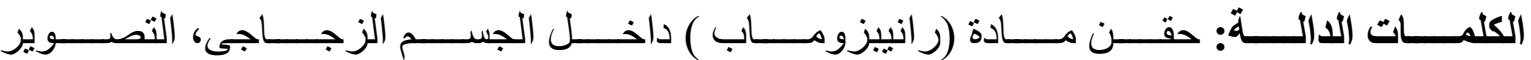
الوعائى المقطعى البصرى المتر ابط، قصر النظر. 